\title{
Compiling a Model of Managers' Professional Meritocra- cy Based on Islamic and Iranian Teachings
}

\section{Compilación de un modelo de meritocracia profesional de geren- tes basado en enseñanzas islámicas e iraníes}

\author{
Azar Taghipour \\ Islamic Azad University, Kazerun, Iran. \\ ORCID: https://orcid.org/0000-0001-8761-8441 \\ Alireza Ghasemizad* \\ Islamic Azad University, Kazerun, Iran. \\ ORCID: https://orcid.org/0000-0003-3099-3384 \\ Hamid Reza Motamed \\ Islamic Azad University, Kazerun, Iran. \\ ORCID: https://orcid.org/0000-0003-3026-2012
}

Received 06-14-20 Revised 08-10-20 Accepted 09-01-20 On line 09-30-20

*Correspondence

Email: alirezaghasemizad@gmail.com
Cite as:

Taghipour, A., Ghasemizad, A., \& Reza Motamed, H. (2020) Compiling a Model of Managers' Professional Meritocracy Based on Islamic and Iranian Teachings. Propósitos y Representaciones, 8 (SPE3), e771. Doi: http://dx.doi.org/10.20511/pyr2020.v8nSPE3.771 


\section{Summary}

The purpose of this study is to develop a model of professional meritocracy of managers based on Iranian and Islamic teachings. The research method is mixed (qualitatively quantitative) of the type of successive exploratory designs. In the qualitative section, using the library method and mining texts, the concepts related to the indicators of professional competence of organizational managers were examined. The statistical population in this section was 47 educational management experts who were selected by purposive sampling method and using the analysis of semi-open interview results and content analysis method, concepts were drawn in the form of a network of themes. The tools for collecting research information in the qualitative section were sources, interviews and documents (research reports, etc.) that the participatory feedback method was used to validate the validity and reliability analysis. In order to analyze the qualitative data, inductive content analysis based on open coding was used. In the quantitative part, data collection was based on a researcher-made questionnaire. In this part, a one-sample t-test was used and the statistical population of this section was 213 And were bred in Bushehr province that multi-stage cluster sampling method was used to select quantitative statistical samples. To validate the reliability of the questionnaire in this section, Cronbach's alpha coefficient was used, the reliability of which was 0.78 , and its face and content validity was confirmed by experts. Data analysis in the qualitative domain was based on content analysis and in the quantitative domain was done using statistical software and factor analysis method. The results showed that the development of human resource management model with qualification criteria in accordance with Islamic and Iranian teachings, organizational planning based on "competence", matching capabilities and competencies with jobs and managements should be done carefully and only under legal and ethical criteria.

Keywords: Organizational Management, Professional Merit, Islamic and Iranian Teachings, Bushehr Province.

\section{Resumen}

El propósito de este estudio es desarrollar un modelo de meritocracia profesional de los gerentes basado en las enseñanzas iraníes e islámicas. El método de investigación es mixto (cualitativamente cuantitativo) del tipo de diseños exploratorios sucesivos. En el apartado cualitativo, utilizando el método bibliotecario y los textos mineros, se examinaron los conceptos relacionados con los indicadores de competencia profesional de los gerentes organizacionales. La población estadística en esta sección fue de 47 expertos en gestión educativa que fueron seleccionados mediante el método de muestreo intencional y utilizando el análisis de los resultados de la entrevista semiabierta y el método de análisis de contenido, los conceptos se dibujaron en forma de una red de temas. Las herramientas para la recolección de información de investigación en el apartado cualitativo fueron fuentes, entrevistas y documentos (informes de investigación, etc.) que se utilizó el método de retroalimentación participativa para validar el análisis de validez y confiabilidad. Para el análisis de los datos cualitativos se utilizó el análisis de contenido inductivo basado en codificación abierta. En la parte cuantitativa, la recolección de datos se basó en un cuestionario elaborado por investigadores. En esta parte, se usó una prueba t de una muestra y la población estadística de esta sección fue de 213 Y fueron criados en la provincia de Bushehr que se utilizó el método de muestreo de conglomerados de etapas múltiples para seleccionar muestras estadísticas cuantitativas. Para validar la confiabilidad del cuestionario de este apartado se utilizó el coeficiente alfa de Cronbach, cuya confiabilidad fue de 0,78 , y los expertos confirmaron su validez de cara y contenido. El análisis de datos en el dominio cualitativo se basó en el análisis de contenido y en el dominio cuantitativo se realizó mediante software estadístico y método de análisis factorial. Los resultados mostraron que el desarrollo de un modelo de gestión de recursos humanos con criterios de calificación de acuerdo con las enseñanzas islámicas e iraníes, la planificación organizativa basada en la "competencia", 
la adecuación de las capacidades y competencias con los puestos y la gestión debe realizarse con cuidado y solo bajo criterios legales y éticos.

Palabras clave: Gestión organizacional, mérito profesional, enseñanzas islámicas e iraníes, provincia de Bushehr.

\section{Introduction}

Undoubtedly, in the age of information and communication influx, the management structure of educational centers is one of the main pillars in the structure of education, the quantitative and qualitative decline of which has a direct impact on the goals and performance of education. The success of the educational programs in any country depends on the existence of educators and managers who have the necessary scientific competencies and job and professional skills, are aware of teaching and learning methods, know the content knowledge and are equipped with the knowledge of classroom management, and using educational resources and evaluation methods (Feldman \& Morinio, 2018). In the turmoil market of the modern period, the increasing progress and productivity of any organization owe to the unique capabilities and competencies of its competent manpower. Competence has been and is the lost and ever ideal of humanity; "Competence is the knowledge, skills and ability to perform effectively and successfully, the competence of which is visible and distinguishes excellent from average performance" (Soderquist, 2010). Succession and training managers in accordance with strategies target the organization in a way that the ability of managers is promoted in line with organizational changes and maturity. "Competence is a combination of explicit and implicit knowledge, behavior and skills, which gives the ability and capacity to perform duties." (Dragandis \& Mentras, 2006).

Indeed, the competence of individuals in an organization emerges organizational competence. "Therefore, the relationship of competency between the individual and the organization is a two-way relationship that, due to the benefit by the organization, should naturally grow from the whole to the part, that is, from the organization to the individual". Organizations need to create a suitable environment for the activity of capable people and the emergence of their competency in the social arena" (Donaldson \& Davis, 1990). "Improving the professional competence of managers is the main condition for the success of the organization. Professional competence equips people against scientific and technological changes and commits them towards learning, training and performance improvement" (Fathi Vajargah \& Mohammad Hadi, 2013).

\section{Materials and Methods}

This research is fundamental-applied in terms of purpose and has a deductive-inductive approach. This research has basically a descriptive-analytical approach and then is of applied researches type according to the objectives of the research as a mixed research method and exploratory approach. Due to the need to conduct research by a qualitative method before the quantitative method, in this research, exploratory design has been used as one of the research strategies in mixed methods. To ensure the validity of the tool in the qualitative section of the research, content validity was used and the questionnaire was confirmed by academic professors and experts in this field. To calculate the reliability, the two methods of retest and intra-subject agreement were used (Soleimani \& Karimi, 2016). The obtained coefficients were $81.3 \%$ and $84.2 \%$, respectively, which indicates that its reliability is acceptable.

1.Qualitative Section: In the qualitative section, the concepts related to the evaluation of meritocracy indicators in the sources are investigated by the method of mining texts and by using theme analysis, interviews and books in the field of meritocracy are extracted and are drawn in the form of themes network. Theoretical study is written by the aid of books and articles by experts about the criteria for selecting competent 
managers in accordance with the Islamic Iranian model and related topics. After the purposeful and semi-structured interview, the content of the interview was analyzed using the theme analysis method, and finally 109 basic themes, 16 inclusive themes, and 5 organizing themes were identified. For this purpose, three types of coding were used, which are: open coding, axial coding and selective coding. The required data were collected through "document investigation" and "interview". In the interview section, a semi-structured interview was conducted with 47 educational high school managers, including 22 women and 25 men. Each interview on average lasted 60 minutes. The study of books and performing interviews were conducted based on the researcher's information saturation. Findings in the interview section and written sources were recorded, and then, based on similarities with the initial and centralized coding; codes were assigned to similar cases and converted to categories that were the main purpose of the research. To ensure the validity of the tool in the qualitative section of the research, content validity was used and the questionnaire was confirmed by academic professors and experts in this field. To calculate the reliability, two methods of retest and intra-subject agreement were used. The obtained coefficients were $81.3 \%$ and $84.2 \%$, respectively, which indicates that its reliability is acceptable. To ensure the validity of the tool in the qualitative section of the research, the opinions of academic professors and experts in this field were used. To calculate the reliability of the retest, among the conducted interviews, several interviews were selected as sample and each of them was coded in a short and specific time interval. The reliability coefficient between the performed coding was obtained $81.3 \%$, which indicates its acceptability.

2.Quantitative Section: In the quantitative section, the required information has been composed of two parts through a researcher-made questionnaire consisting of closed questions, that at first the contextual questions and related to the personal characteristics of the respondents are used and then the standard meritocracy questionnaire which consists of 31 items is used. In order to determine the validity of the questionnaire, faced validity, content validity and construct validity were used. In the faced validity, the questionnaires before distribution by the researcher were examined by several sample members and some academic experts. In the content validity in the form of a Delphi method and by the aid of CVR and CVI forms and with the help of ten experts including interviewed members, academic experts, several subjects, and so on, the content of the questionnaire in terms of additional questions and/or the correction of questions was investigated. The CVI form showed that all the questions of professional competence and its influential factors have a proper condition in terms of simplicity, clarity and relevance (the ratio of this coefficient for each one of the questions was higher than 0.79); also, since the CVR value for all questions was obtained above 0.62 , no question needed to be deleted. Regarding construct validity, two types of convergent and divergent validity by the aid of Smart-Pls 2 software were also used. In investigating convergent validity, the findings showed that the significance coefficients of all factor loads were greater than 2.58 (T-statistic), meaning that all factor loads were significant with $99 \%$ confidence; the values of all factor loads were also above 0.5 (explicit and hidden variable relationship); the Average Variance Extracted (AVE) of all components was above 0.5 and also the composite reliability of all components was greater than its average variance extracted; therefore, it can be said that the convergent validity of the model constructs is confirmed. In investigating divergent validity, the Fornell and Larcker test (this test measures divergent validity (diagnostic) at the level of hidden variables using a module defined in Smart-Pls 2 software) and cross loadings test (this test measures divergent validity at the level of observable variables by a module defined in Smart-P1s2 software) were used. In Fornell and Larcker test, the findings showed that the root mean of the extracted variance of each hidden variable was greater than the maximum correlation of that variable with other hidden variables of the model; the results of cross loadings test showed that the factor loads of each of the research variables were higher than the observable factor loads of other measurement models available in the model and on the other hand the factor load of each observable variable on the corresponding hidden variable was at least 0.1 higher than the factor loads of the same observa- 
ble variable on other hidden variables. Therefore, the results of these two tests indicated divergent validity (Ghirshman, 2016).

In this research, reliability was calculated through Cronbach's alpha and composite reliability. The values of these two coefficients for all research variables were obtained above 0.7, which indicates the reliability of the measurement tool. The reliability and validity coefficients mentioned for the questionnaire of the professional competence of managers and the factors affecting it are confirmed (Mohammadi et al., 2013). Because Cronbach's alpha and composite reliability coefficient are above 0.7 and also AVE $>0.5$. Convergent validity is confirmed, because $\mathrm{CR}>0.7$; $\mathrm{CR}>\mathrm{AVE}$; AVE> 0.5 and divergent validity is also confirmed, because MSV $<\mathrm{AVE}$ and ASV $<\mathrm{AVE}$.

The statistical population includes all high school managers in Bushehr province and the statistical sample size was determined using Cochran's formula equal to 203 people. In order to achieve the considered statistical sample, stratified sampling method was used. The statistical population is divided into two classes of Bushehr province high school managers and educational experts and specialists and a sample was selected from each class according to the size of the statistical population. First, the cities of Bushehr province that have the highest population in relation to the whole province were identified and the cities of Bushehr, Borazjan, Bandar Genaveh, Bandar Kangan and Khormuj participated in the study. This sampling was considered as the first cluster. In the next stage, the high schools of these cities were identified and selected from each city in proportion to the school community of girls and boys, and finally the number of educational managers was randomly selected (Geejung \& Keelee, 2020).

\section{Results}

In the analysis of the findings of the qualitative section, the method of theme analysis was used. The theme or content expresses the concept of the pattern available in the data and is related to the research questions. The results were obtained through interviews and coding of concepts (Group, 2003). After analyzing the major and minor questions of the research, it became clear that the mechanisms, facilitators and obstacles in the field of designing and compiling the professional criteria of elite selection and the method of selecting specialized people and competent employees in organizational management, in accordance with Iranian and Islamic teachings and trainings will definitely help greatly organizing human resource management and professional ethics excellence (Kimmer et al., 2017). Information analysis in thematic analysis method is based on the coding process. This method is a process for analyzing textual data that converts scattered and diverse data into rich and detailed data (Sobhaninejad \& Tejdan, 2015). Information analysis based on a specific procedure systematizes the following themes in four stages: "seeing the text", "proper perception and understanding of seemingly irrelevant information", "analysis of qualitative information" and finally "systematic observation of a person, interaction, group, situation, organization or culture". The basic themes include the codes and key points available in the text. The organizing themes include the categories obtained from the combination and summary of the basic themes and the inclusive themes or main themes include the principles governing the text as a whole. Then, these themes are drawn as a network of themes in which the prominent themes of each of the three mentioned levels, along with the relationships between them have been shown (Abedi Jafari et al., 2011). (Table 1) contains 109 basic themes obtained from the analysis of interviews and written sources in this field, which are: Quran, Nahjul-Balagha, History of Education in Islam and Iran by Mohammad Ali Almasi and Iran from the Beginning to Islam by Roman Ghirshman. 


\section{Table 1.}

Basic Themes Obtained from Interviews and Written Sources in This Field

\section{Basic Themes}

\begin{tabular}{|c|c|c|c|c|c|}
\hline & $\begin{array}{c}\text { The Holy Quran } \\
\text { Page(s) }\end{array}$ & $\begin{array}{l}\text { Nahjul- } \\
\text { Balagha } \\
\text { Page(s) }\end{array}$ & $\begin{array}{l}\text { Inter- } \\
\text { view } \\
\text { Page(s) }\end{array}$ & $\begin{array}{l}\text { History of Edu- } \\
\text { cation in Islam } \\
\text { and Iran Author: } \\
\text { Ali Almasi }\end{array}$ & $\begin{array}{l}\text { Iran from the Be- } \\
\text { ginning to Islam } \\
\text { by Roman } \\
\text { Ghirshman }\end{array}$ \\
\hline $\begin{array}{l}\text { Having expertise } \\
\text { and experience in } \\
\text { adopting the ap- } \\
\text { propriate man- } \\
\text { agement style }\end{array}$ & $\begin{array}{l}\text { Anam (124) } \\
\text { Saad (46) }\end{array}$ & & $\bullet$ & $\begin{array}{c}221,280,216,303 \\
, 223,316,319 \\
322,433,439\end{array}$ & - \\
\hline $\begin{array}{l}\text { Management of } \\
\text { organizational } \\
\text { budgets and } \\
\text { credits }\end{array}$ & & $\begin{array}{l}\text { Letter } \\
24\end{array}$ & $\bullet$ & & \\
\hline $\begin{array}{l}\text { Ability to ana- } \\
\text { lyze the manage- } \\
\text { rial conditions } \\
\text { and requirements } \\
\text { of the organiza- } \\
\text { tion }\end{array}$ & & & & $\begin{array}{c}197,200,218,221 \\
, 224, \\
291,345,434\end{array}$ & \\
\hline $\begin{array}{l}\text { Intellectual ca- } \\
\text { pability to guide } \\
\text { the affairs, tools } \\
\text { and environment } \\
\text { of the organiza- } \\
\text { tion }\end{array}$ & & & & $\begin{array}{c}10,11,48,64,67,7 \\
172,73,30,75,76, \\
168,90,116, \\
121,178,143, \\
170,161\end{array}$ & $\begin{array}{c}34,65,34,327, \\
152,181,214, \\
290,365,389, \\
347,350,352, \\
355,356,359,4, \\
56,82,328,345\end{array}$ \\
\hline $\begin{array}{l}\text { Crisis manage- } \\
\text { ment and unex- } \\
\text { pected affairs in } \\
\text { the organization }\end{array}$ & $\begin{array}{c}\text { Kauther } 7 \text { (12) } \\
\text { Dahr } 24 \text { Balad } \\
17\end{array}$ & & & $\begin{array}{l}200,218,219 \\
188,222,321 \\
400\end{array}$ & \\
\hline $\begin{array}{l}\text { Having capable } \\
\text { human resources } \\
\text { to advance goals }\end{array}$ & & & $\bullet$ & $\begin{array}{c}11,79,86,87,130 \\
147,67\end{array}$ & $\begin{array}{c}39,183,313,327 \\
390,329,338\end{array}$ \\
\hline $\begin{array}{l}\text { Forming a strong } \\
\text { advisory team to } \\
\text { carry out partner- } \\
\text { ship management } \\
\text { affairs }\end{array}$ & $\begin{array}{l}\text { Al Imran } 159 \\
\text { Yusuf } 80 \text { Shoo- } \\
\text { raa } 38 \text { A'raf 198, } \\
\text { Fussilat } 5\end{array}$ & & $\bullet$ & $\begin{array}{c}327,400,327 \\
389\end{array}$ & \\
\hline $\begin{array}{l}\text { Operational ca- } \\
\text { pability in using } \\
\text { the promotion } \\
\text { opportunities of } \\
\text { the organization }\end{array}$ & & & $\bullet$ & & \\
\hline $\begin{array}{l}\text { Ability to coor- } \\
\text { dinate between } \\
\text { organizational } \\
\text { needs and de- } \\
\text { mands }\end{array}$ & & & & $\begin{array}{c}31,97,159,160, \\
165\end{array}$ & \\
\hline $\begin{array}{l}\text { Professional } \\
\text { planning for bal- } \\
\text { anced develop- } \\
\text { ment of the or- }\end{array}$ & & & $\bullet$ & $\begin{array}{c}66,73,113,164,9 \\
15,68,96,98 \\
157,161,163, \\
166,167\end{array}$ & $\begin{array}{c}13,22,67,75, \\
182,165,174, \\
243,252,293, \\
314,336,99,113,\end{array}$ \\
\hline
\end{tabular}

\section{Source:}




\begin{tabular}{|c|c|c|c|c|c|}
\hline ganization & & & & & $\begin{array}{c}173,328,338,15 \\
19,261\end{array}$ \\
\hline $\begin{array}{l}\text { Planning in order } \\
\text { to select compe- } \\
\text { tent organiza- } \\
\text { tional forces and } \\
\text { managerial suc- } \\
\text { cession }\end{array}$ & $\begin{array}{c}11 \text { Baqarah } 247 \\
\text { Nisa An'am } \\
\text { 165/86 Anfal } \\
\text { 75/34 Tawbah } \\
\text { Anbiya } \\
\text { 51/72/7/105 } \\
\text { Hajj 23 - } \\
\text { Mu'minūn11 } \\
\text { Nur 55 Naml 15 } \\
\text { Qasas } 7 \text { Saaffaat } \\
\text { 112/160 } \\
\text { Ankabut 9/27 } \\
\text { Saad 47 Zukhruf } \\
\text { 32 Dukhan 32 } \\
\text { Jathiyah 16 Mu- } \\
\text { jādilah 11 Fajr } \\
\text { 29 Inshirah } 4\end{array}$ & & $\bullet$ & $\begin{array}{c}200,292,305, \\
425\end{array}$ & \\
\hline $\begin{array}{l}\text { Forming special } \\
\text { teams to quickly } \\
\text { organize and ad- } \\
\text { vance the affairs } \\
\text { of the organiza- } \\
\text { tion }\end{array}$ & $\begin{array}{c}\text { 10 Baqaarah } \\
\text { 220/224 Nisa } \\
\text { 114/146 Mā’idah } \\
\text { 39/154 / A'raf } \\
\text { 142 Hud 88 Nahl } \\
\text { 119 Isra25 } \\
\text { Anbiya 90 Nur 5 } \\
\text { Ahzab 71 Shoo- } \\
\text { raa 40 Muham- } \\
\text { mad 2/5 Dukhan } \\
\text { 19 Najm } 32 \\
\text { Naazi'aat } 5 \\
\text { Hadid 23 Nisa } \\
\text { 32 An'am } 142 \\
\text { A'raf } 19\end{array}$ & & $\bullet$ & $\begin{array}{c}338,209,432, \\
433\end{array}$ & \\
\hline $\begin{array}{l}\text { Resource opti- } \\
\text { mization, design- } \\
\text { ing and imple- } \\
\text { menting man- } \\
\text { agement pro- } \\
\text { cesses }\end{array}$ & $\begin{array}{c}\text { 56 Rum } 5 \text { Ahzab } \\
\text { 25/21 Saba } 27 \\
\text { Ghafir } 42 \text { Dāri- } \\
\text { yāt } 58 \text { Burooj } 15\end{array}$ & & $\bullet$ & $\begin{array}{l}260,340,356, \\
386,416,424\end{array}$ & \\
\hline $\begin{array}{l}\text { Creating special } \\
\text { opportunities and } \\
\text { sustainable com- } \\
\text { petitive ad- } \\
\text { vantage for the } \\
\text { organization }\end{array}$ & $\begin{array}{l}81 \text { Fath } 29 \bar{A} 1 \\
\text { 'Imrān } \\
\text { 103/200/3 Shoo- } \\
\text { raa42/38 Tawbah } \\
122 \text { Mă'idah } 2\end{array}$ & & & 26,144 & $\begin{array}{l}71,78,81,106, \\
111,296,302, \\
397,391,371, \\
354,106,343\end{array}$ \\
\hline $\begin{array}{l}\text { Utilizing trained } \\
\text { and experienced } \\
\text { human resources } \\
\text { in the middle } \\
\text { management } \\
\text { body of the or- } \\
\text { ganization }\end{array}$ & & & $\bullet$ & $\begin{array}{c}21,25,43,54 \\
115,113,119, \\
120,117,134, \\
163,165,166, \\
170,172\end{array}$ & $\begin{array}{c}43,73,78,111 \\
140,151,172 \\
255,258,334 \\
335,397,402\end{array}$ \\
\hline $\begin{array}{l}\text { Appointment of } \\
\text { specialized and }\end{array}$ & $\begin{array}{l}\text { Yusuf } 55 \text { Qasas } \\
25,26 \text { Nisa } 58\end{array}$ & Letter 4 & $\bullet$ & $\begin{array}{c}296,345,366, \\
425\end{array}$ & \\
\hline
\end{tabular}


committed young

human resources

in the organiza-

tion

Familiarity with the strengths and

Baqarah44

weaknesses of

27/28 Mā'idah

the school and

planning based

on existing reali-

ty

Individual and

Nisa 139 Yunus

173, 171, 32,

65 Fath 3

social piety and

Baqarah 168

$372,349,352$,

chastity during

the tenure

Mā'idah 75,56

367,404

Tawbah 108 p.

26 Naziat 40

Alaa 14

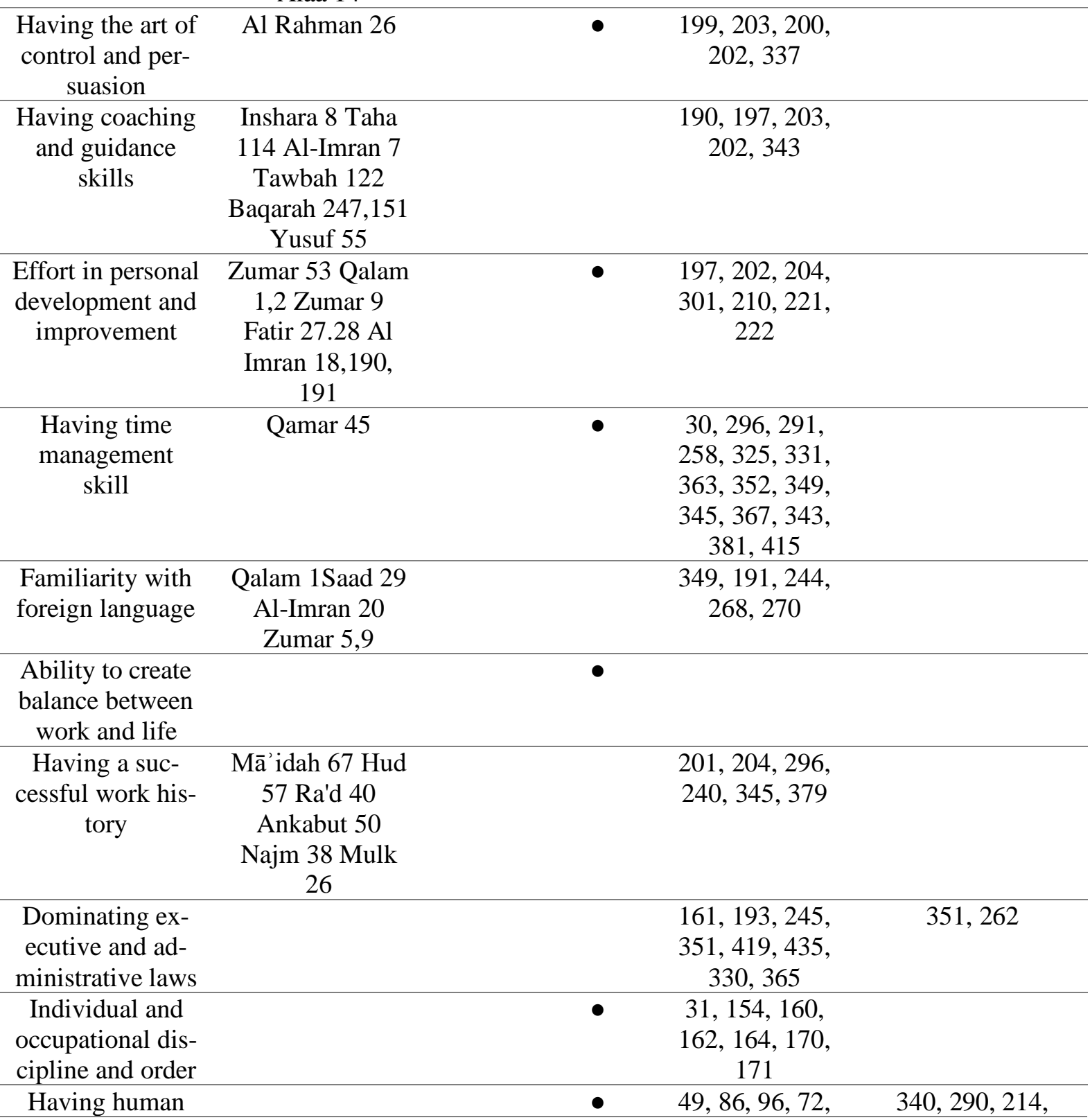




\begin{tabular}{|c|c|c|c|c|c|}
\hline $\begin{array}{l}\text { virtues and posi- } \\
\text { tive managerial } \\
\text { authority }\end{array}$ & & & & $\begin{array}{l}120,141,152, \\
162,165,171, \\
173\end{array}$ & $\begin{array}{c}181,139,308, \\
286,139,100,76, \\
345,391,353, \\
352,349,338\end{array}$ \\
\hline $\begin{array}{l}\text { Having commit- } \\
\text { ment and con- } \\
\text { science and ob- } \\
\text { serving public } \\
\text { rights in the or- } \\
\text { ganization }\end{array}$ & $\begin{array}{c}\text { Baqarah 250, } \\
177,155,153, \\
\text { Al Imran 200, } \\
\text { 146, 142, 140, } \\
25 \text { A'raf } 137,95 \\
\text { Hud 112, Ra'd } \\
\text { 24 Luqman } 31, \\
\text { 17 Ahzab } 21 \\
\text { Shooraa } 15 \\
\text { Luqman } 31,17\end{array}$ & & $\bullet$ & & \\
\hline $\begin{array}{l}\text { Legalism with } \\
\text { the focus on } \\
\text { moral commit- } \\
\text { ment and admin- } \\
\text { istrative equality }\end{array}$ & & $\begin{array}{c}\text { Letter } \\
76\end{array}$ & & $\begin{array}{l}49,86,96,72, \\
120,141,152, \\
162,165,171, \\
173\end{array}$ & $\begin{array}{c}76,100,139,286 \\
308,139,181, \\
214,290,341,345\end{array}$ \\
\hline $\begin{array}{l}\text { Tendency to be } \\
\text { normative and } \\
\text { follow organiza- } \\
\text { tional rules }\end{array}$ & $\begin{array}{c}\text { Mā'idah 87, } 78 \\
\text { A'raf } 19 \text { Yusuf } \\
79 \text { Yasin 40 Fatir } \\
\text { 36 Rahman } 30\end{array}$ & & $\bullet$ & 204,292 & \\
\hline $\begin{array}{l}\text { Administrative } \\
\text { discipline and } \\
\text { balance of organ- } \\
\text { izational affairs } \\
\text { management }\end{array}$ & & $\begin{array}{c}\text { Letter } \\
17\end{array}$ & $\bullet$ & & \\
\hline $\begin{array}{l}\text { Development of } \\
\text { professional eth- } \\
\text { ics and respect } \\
\text { and communica- } \\
\text { tion with the cli- } \\
\text { ent }\end{array}$ & & & & $\begin{array}{l}49,86,96,72, \\
120,141,152, \\
162,165,171, \\
173\end{array}$ & $\begin{array}{c}76,100,139,286, \\
308,139,181, \\
214,290,338, \\
349,352,353,391\end{array}$ \\
\hline $\begin{array}{l}\text { Compiling pro- } \\
\text { fessional ethics } \\
\text { in the manage- } \\
\text { ment regulations } \\
\text { of Iranian organ- } \\
\text { izations }\end{array}$ & $\begin{array}{l}\text { Al Imran 103, } \\
\text { 199 Nisa } 9 \text { Anfal } \\
63 \text { Naml } 61\end{array}$ & & $\bullet$ & $387,386,400$ & \\
\hline $\begin{array}{l}\text { Paying attention } \\
\text { to the problems } \\
\text { of employees and } \\
\text { students and em- } \\
\text { pathizing with } \\
\text { them }\end{array}$ & $\begin{array}{c}\text { Noor } 27 \text { Isra } 23 \\
\text { Maryam } 14 \\
\text { Ankabut } 8 \\
\text { Luqman } 14 \\
\text { Ahqaf } 15\end{array}$ & & & $\begin{array}{c}344,349,353 \\
358\end{array}$ & \\
\hline $\begin{array}{l}\text { Mastery of inter- } \\
\text { active manage- } \\
\text { ment techniques } \\
\text { in the organiza- } \\
\text { tional field }\end{array}$ & $\begin{array}{c}\text { Baqarah } 274, \\
277 \text { Nisa } 99,104 \\
\text { Mā'idah } 184 \\
\text { A'raf 56,129 } \\
\text { Tawbah 6,102 } \\
\text { Hud 62 Yusuf } \\
\text { 62,83,87 Hijr 55 } \\
\text { Isra 8,57 Kahf }\end{array}$ & & $\bullet$ & & \\
\hline
\end{tabular}


40,110 Maryam

18,48 Anbiya 90

Mu'minūn 100

Naml 72 Qasas 9

Yasin 74

Ankabut 5,36

Rum 24 Sajda 16

\begin{tabular}{|c|c|c|c|c|}
\hline $\begin{array}{l}\text { Capable to apply } \\
\text { new methods of } \\
\text { communication } \\
\text { skills }\end{array}$ & $\begin{array}{c}\text { Baqarah 261, } \\
\text { Ma'idah 99, } \\
\text { An'am 59, 73, } \\
\text { 80, 103, A'raf 7, } \\
\text { 62 Anfal 47, 75 } \\
\text { Tawbah 16, 78 } \\
\text { Yunis 61 Hud } \\
\text { 123 R'ad 8 Ibra- } \\
\text { him } 38 \text { Nahl 19 } \\
\text { Kahf 45 Maryam } \\
\text { 64, } 94 \text { Ta-Ha 7, } \\
\text { 98 Haj 75, 76 } \\
\text { Mu'minūn 88 } \\
\text { Zumar 7, Qafar } \\
\text { 19, Fusșilat 54 } \\
\text { Shooraa 12 Fath } \\
\text { 21 Hujurat 18, } \\
\text { Hadid 3, 4, Talaq } \\
\text { 12 Jinn 28 }\end{array}$ & $\bullet$ & $\begin{array}{l}199,212,296, \\
328,332,344, \\
339,347,358, \\
406,426,431\end{array}$ & \\
\hline $\begin{array}{l}\text { Maintaining and } \\
\text { strengthening a } \\
\text { wide range of } \\
\text { audiences inside } \\
\text { and outside the } \\
\text { organization }\end{array}$ & & $\bullet$ & $22,30,48$ & 401 \\
\hline $\begin{array}{l}\text { The need for a } \\
\text { structural rela- } \\
\text { tionship between } \\
\text { the organization } \\
\text { and the perfor- } \\
\text { mance of manag- } \\
\text { ers }\end{array}$ & & $\bullet$ & & \\
\hline $\begin{array}{c}\text { Continuous } \\
\text { communication } \\
\text { of managers with } \\
\text { the world of cul- } \\
\text { ture and art for } \\
\text { the environmen- } \\
\text { tal effectiveness } \\
\text { of the organiza- } \\
\text { tion }\end{array}$ & & & 166 & $\begin{array}{l}156,167,308, \\
323,169,193, \\
205,277,323\end{array}$ \\
\hline $\begin{array}{l}\text { Mission to sup- } \\
\text { port the commu- } \\
\text { nication skills of } \\
\text { the managers of } \\
\text { the organization }\end{array}$ & & & $\begin{array}{c}11,41,48,50 \\
78,82,84,100 \\
131,174\end{array}$ & $\begin{array}{c}3,77,36,100, \\
294,295,363, \\
399,157,243, \\
259,294\end{array}$ \\
\hline $\begin{array}{l}\text { Interaction with } \\
\text { the central organ- }\end{array}$ & & $\bullet$ & $132,160,177$ & $\begin{array}{c}56,62,65,74,82 \\
115,133,100\end{array}$ \\
\hline
\end{tabular}




\begin{tabular}{|c|c|c|c|c|}
\hline $\begin{array}{l}\text { ization and other } \\
\text { relevant organi- } \\
\text { zations }\end{array}$ & & & & $\begin{array}{c}179,226,309 \\
115,399,294 \\
296,309,326, \\
360,352,351 \\
350,343,340,341\end{array}$ \\
\hline $\begin{array}{l}\text { Utilizing trained } \\
\text { and experienced } \\
\text { human resources } \\
\text { in the middle } \\
\text { management } \\
\text { body of the or- } \\
\text { ganization }\end{array}$ & & $\bullet$ & $\begin{array}{c}20,22,42,72, \\
160,164,165, \\
167,171,66, \\
121\end{array}$ & $50,309,363,401$ \\
\hline $\begin{array}{l}\text { Having the art of } \\
\text { attracting the } \\
\text { audience and } \\
\text { organizational } \\
\text { management } \\
\text { techniques }\end{array}$ & $\begin{array}{c}\text { Baqarah 259 } \\
\text { Mā'idah 75 } \\
\text { An'am 57 Anbi- } \\
\text { ya } 24 \text { Naml } 64 \\
\text { Rum 35/ 58/ 47/ } \\
\text { 37 Ghāfir } \\
\text { Zakhraf 21/36, } \\
\text { Jasieh 4/6, Hadid } \\
\text { 17/25, Taghabun } \\
6\end{array}$ & $\bullet$ & & $\begin{array}{c}200,207,209 \\
364,379,213 \\
217,246,393 \\
409,423\end{array}$ \\
\hline $\begin{array}{l}\text { Having grand } \\
\text { nature against } \\
\text { opponents and } \\
\text { opposing opin- } \\
\text { ions }\end{array}$ & $\begin{array}{c}\text { Baqarah 177/ } \\
\text { 249/235/45 Al } \\
\text { Imran 146/ 200/ } \\
\text { 186/ 120/ 17 } \\
\text { An'am 34 A'raf } \\
\text { 128/ 126/ 87 An- } \\
\text { fal 66/46 Taw- } \\
\text { bah 114 Yusuf } \\
\text { 90/ 83/ 18 R'ad } \\
\text { 21 Ibrahim 5/12 } \\
\text { Nisa 25 } \\
\text { Mā'idah101 } \\
\text { Yunis 109 Hud } \\
\text { 87/49/11 Nahl } \\
\text { 127/ 126/110/ } \\
\text { 96/ 42 Isra } \\
\text { 106/44f } \\
\text { 69/68/67/ 28 } \\
\text { Maryam 65 Ta- } \\
\text { Ha 132/ 111/ 30/ } \\
\text { 25 Anbiya 85 } \\
\text { Haj 59/35 } \\
\text { Mu'minūn111 } \\
\text { Saffat 102/101 } \\
\text { Ankabut 59 Rum } \\
\text { 60 Luqman 17 } \\
\text { Sajda 24 Ahzab } \\
\text { 51/ 35/21 Saba } \\
\text { 19 Fatir 41 Saad } \\
\text { 17/43 Zumar } \\
\text { 22/10 Ghafir } \\
\text { 77/55 Shooraa } \\
\text { 43/33 Muham- }\end{array}$ & & 244,390 & \\
\hline
\end{tabular}


mad 31 Hujurat

5 Taghabun 17

Qalam 48 Muz-

zammil 10

\begin{tabular}{|c|c|c|c|c|c|}
\hline $\begin{array}{l}\text { Having proper } \\
\text { and respectful } \\
\text { relations with } \\
\text { colleagues }\end{array}$ & & $\begin{array}{l}\text { Letter } \\
76\end{array}$ & & $\begin{array}{c}22,23,27,48 \\
50,51,84,68 \\
85,72,75,100 \\
152,159,162 \\
171\end{array}$ & $\begin{array}{c}304,308,314 \\
358,386\end{array}$ \\
\hline $\begin{array}{l}\text { Organizational } \\
\text { conflicts man- } \\
\text { agement }\end{array}$ & $\begin{array}{c}\text { Baqaarah } 174 \mathrm{Al} \\
\text { Imran } 134 \mathrm{~A} \text { 'raf } \\
20 \text { Haj } 67,68\end{array}$ & & & 403,404 & \\
\hline $\begin{array}{l}\text { Thinking togeth- } \\
\text { er and attention } \\
\text { to the principle } \\
\text { of consultation }\end{array}$ & $\begin{array}{l}\text { Yusuf } 80 \text { Shoo- } \\
\text { raa } 38 \text { A'raf } 198 \\
\text { Fușșilat } 5 \text { Al } \\
\text { Imran } 159\end{array}$ & & & $\begin{array}{c}22,98,139,141 \\
142,143,1,46 \\
138,137\end{array}$ & $\begin{array}{c}88,114,313,355, \\
279,336,132, \\
176,241,251 \\
308,338,340\end{array}$ \\
\hline $\begin{array}{l}\text { Not interfering } \\
\text { cultural and eth- } \\
\text { nic distinctions }\end{array}$ & & & & $162,171,174$ & \\
\hline $\begin{array}{l}\text { Applying suc- } \\
\text { cessful experi- } \\
\text { ences of other } \\
\text { cultures }\end{array}$ & & & & & $\begin{array}{l}150,179,183 \\
255,265,363\end{array}$ \\
\hline $\begin{array}{l}\text { Cultural study } \\
\text { and cultural pa- } \\
\text { thology }\end{array}$ & & & $\bullet$ & $\begin{array}{c}20,23,28,85 \\
174\end{array}$ & 319,402 \\
\hline $\begin{array}{l}\text { Competence to } \\
\text { work with di- } \\
\text { verse cultures }\end{array}$ & & & & $29,80,99,175$ & $\begin{array}{c}391,397,406 \\
407,409\end{array}$ \\
\hline $\begin{array}{l}\text { Creative use of } \\
\text { project manage- } \\
\text { ment knowledge } \\
\text { in the organiza- } \\
\text { tion }\end{array}$ & $\begin{array}{c}\text { Mā’idah 17 A'raf } \\
54 \text { Yunis 4/6 } \\
\text { Hud } 7 \text { Ibrahim } \\
\text { 32/19 Hijr 86/92 } \\
\text { Nahl 13 Haj 5 } \\
\text { Noor 45 Shu'ara } \\
\text { 184 Yasin 36 } \\
\text { Ankabut 20/44 } \\
\text { Rum 19/20 Sajda } \\
\text { 4/7 Wāqi 'ah 35 } \\
\text { Hadid } 4 \text { Talaq } 12 \\
\text { Q̈iyamah 38A'laa } \\
\text { 2 Tin 4 }\end{array}$ & & $\bullet$ & $\begin{array}{c}363,376,381 \\
389,396\end{array}$ & $\begin{array}{c}300,243,288 \\
275,350,379 \\
364,416,421,422\end{array}$ \\
\hline $\begin{array}{l}\text { Ability to flour- } \\
\text { ish creativities } \\
\text { and manifesting } \\
\text { individual oppor- } \\
\text { tunities }\end{array}$ & $\begin{array}{c}\text { Haj } 5 \text { An'am } 152 \\
\text { A'raf } 42\end{array}$ & & $\bullet$ & 203 & \\
\hline $\begin{array}{l}\text { Increasing moti- } \\
\text { vation, dyna- } \\
\text { mism, creativity, } \\
\text { innovation, and } \\
\text { mobility of man- } \\
\text { agers }\end{array}$ & & & $\bullet$ & $\begin{array}{c}15,22,13,114 \\
164,173\end{array}$ & $142,254,295$ \\
\hline Ability to pro- & & Letter 7 & $\bullet$ & & \\
\hline
\end{tabular}




\begin{tabular}{|c|c|c|c|c|}
\hline \multicolumn{5}{|l|}{$\begin{array}{c}\text { duce new } \\
\text { thoughts and ide- } \\
\text { as }\end{array}$} \\
\hline $\begin{array}{l}\text { Guiding teachers } \\
\text { to improve the } \\
\text { quality of the } \\
\text { teaching-learning } \\
\text { process }\end{array}$ & & & $\begin{array}{c}51,136,144 \\
158\end{array}$ & $\begin{array}{c}101,294,90,277 \\
336\end{array}$ \\
\hline $\begin{array}{l}\text { Having the abil- } \\
\text { ity for adaptive } \\
\text { and innovative } \\
\text { thinking }\end{array}$ & & $\bullet$ & $\begin{array}{l}233,234,236 \\
286,260,273\end{array}$ & \\
\hline $\begin{array}{l}\text { Having problem } \\
\text { solving skill }\end{array}$ & $\begin{array}{l}\text { Nisa } 94 \text { A'raf } \\
154 \text { Hujurat } 6\end{array}$ & & $245,308,409$ & \\
\hline $\begin{array}{l}\text { Having a search } \\
\text { spirit }\end{array}$ & $\begin{array}{c}\text { Baqarah } \\
\text { 259/122/75 Al } \\
\text { Imran 64/19 } \\
\text { An'am 57 A'raf } \\
105 \text { Yusuf 51 } \\
\text { Isra 5 Haj 31/6 } \\
\text { Muminun 62 } \\
\text { Rum 52 Sajda } 3 \\
\text { Ahzab 70/4 Saba } \\
\text { 20/ 23 Fatir 24 } \\
\text { Ahzab 8 Saad } \\
\text { 284/6 Zumar } \\
\text { 4/41 Ghafir 25 } \\
\text { Fath 27 Dāriyāt } \\
\text { 23 Dahr 3 }\end{array}$ & & $245,308,409$ & \\
\hline $\begin{array}{l}\text { Optimization, } \\
\text { design and de- } \\
\text { velopment of } \\
\text { middle manage- } \\
\text { ment systems }\end{array}$ & $\begin{array}{l}\text { Rahman } 2 \text { Alaq } \\
\text { 4/5 Zumar } 9 \text { Do- } \\
\text { khan } 14 \text { Kahf } 65 \\
\text { Nahl } 27 \text { Baqarah } \\
\text { 151/ 129/251 }\end{array}$ & $\bullet$ & $\begin{array}{l}188,189,206, \\
201,236,211, \\
294,246,256, \\
215,127,230\end{array}$ & \\
\hline $\begin{array}{l}\text { Creating Trans- } \\
\text { formation }\end{array}$ & $\begin{array}{c}\text { Alaq 4/5 Tawbah } \\
122 \text { A'raf } 154 \\
\text { Rahman } 2 \text { Huju- } \\
\text { rat6 Kahf } 65\end{array}$ & $\bullet$ & 234,246 & \\
\hline $\begin{array}{l}\text { Paying attention } \\
\text { to the quality of } \\
\text { educational envi- } \\
\text { ronment facilities }\end{array}$ & An'am 97/98 & $\bullet$ & 435,437 & \\
\hline $\begin{array}{l}\text { Planning for } \\
\text { qualitative im- } \\
\text { plementation of } \\
\text { extracurricular } \\
\text { and educational } \\
\text { complementary } \\
\text { activities }\end{array}$ & $\begin{array}{c}\text { Anbiya 51,10 } \\
\text { Luqman } 17 \mathrm{Haj} \\
5 \text { Qasas } 14 \\
\text { Baqaah } 286 \\
\text { Ma'idah } 48 \mathrm{Al} \\
\text { Imran } 61 \mathrm{Hud} \\
11,18 \text { Waqia } 7 \\
\text { Jinn 11 Sajda } 18\end{array}$ & $\bullet$ & & \\
\hline $\begin{array}{l}\text { Promoting envi- } \\
\text { ronmental attrac- } \\
\text { tiveness }\end{array}$ & & $\bullet$ & & $294,319,396,408$ \\
\hline
\end{tabular}




\begin{tabular}{|c|c|c|c|c|}
\hline $\begin{array}{l}\text { Familiarity with } \\
\text { combined meth- } \\
\text { ods of human } \\
\text { resource support }\end{array}$ & & & $\begin{array}{c}81,85,95,140 \\
78\end{array}$ & $\begin{array}{c}28,70,128,10, \\
140,145,250, \\
225,263,343, \\
358,305,343, \\
345,360,363,397\end{array}$ \\
\hline $\begin{array}{l}\text { Obtaining inter- } \\
\text { national profes- } \\
\text { sional standard } \\
\text { certifications }\end{array}$ & & $\bullet$ & & \\
\hline $\begin{array}{l}\text { Interdisciplinary } \\
\text { science and } \\
\text { knowledge }\end{array}$ & & $\bullet$ & 231 & $\begin{array}{c}156,228,283 \\
313,328\end{array}$ \\
\hline $\begin{array}{l}\text { Knowledge of } \\
\text { Behavioral Psy- } \\
\text { chology and Ed- } \\
\text { ucational Scienc- } \\
\text { es }\end{array}$ & & $\bullet$ & $\begin{array}{c}151,159,160 \\
139,162,138 \\
152,151,162 \\
168,172\end{array}$ & $\begin{array}{c}61,100,140,306 \\
330,329,394 \\
363,402,306 \\
329,405,408 \\
352,363,364\end{array}$ \\
\hline $\begin{array}{l}\text { Ability to dis- } \\
\text { tribute } \\
\text { knowledge on } \\
\text { time }\end{array}$ & & & $\begin{array}{c}12,24,26,51 \\
96,67,87,158 \\
159,169,174 \\
180\end{array}$ & $\begin{array}{c}387,395,340 \\
404,406\end{array}$ \\
\hline $\begin{array}{l}\text { Ability to en- } \\
\text { courage the crea- } \\
\text { tion of new } \\
\text { knowledge }\end{array}$ & & $\bullet$ & $\begin{array}{c}39,54,55,47 \\
58,70,90,113, \\
114,115,116, \\
132,120,157\end{array}$ & $\begin{array}{c}88,97,294,319 \\
396,311,408\end{array}$ \\
\hline $\begin{array}{l}\text { Updating } \\
\text { knowledge re- } \\
\text { sources }\end{array}$ & Ma'idah67 & & $\begin{array}{l}245,255,280 \\
\quad 401,405\end{array}$ & \\
\hline $\begin{array}{l}\text { Utilizing the } \\
\text { capacity of the } \\
\text { media in direct- } \\
\text { ing organization- } \\
\text { al affairs }\end{array}$ & & & $66,73,113,164$ & $\begin{array}{c}67,75,182,165 \\
174,243,252, \\
336,338,328 \\
113,173,261\end{array}$ \\
\hline $\begin{array}{c}\text { Benefit from new } \\
\text { mass media } \\
\text { techniques }\end{array}$ & & & $\begin{array}{c}9,15,94,96 \\
157\end{array}$ & $\begin{array}{c}98,58,109,145 \\
282,382,151 \\
246,294\end{array}$ \\
\hline $\begin{array}{l}\text { Attention to the } \\
\text { international lit- } \\
\text { eracy and media } \\
\text { of managers in } \\
\text { the organization }\end{array}$ & & & $\begin{array}{c}9,11,24,28,57 \\
75,142,157\end{array}$ & $\begin{array}{c}72,80,35,36 \\
345,365,217 \\
222,36,149,345\end{array}$ \\
\hline $\begin{array}{c}\text { Literacy of } \\
\text { working with } \\
\text { new media }\end{array}$ & & $\bullet$ & $\begin{array}{c}9,16,27,47,51 \\
55,115,158 \\
169,165,378\end{array}$ & $\begin{array}{c}67,112,352,379 \\
252\end{array}$ \\
\hline $\begin{array}{l}\text { Using scientific } \\
\text { support and re- } \\
\text { search team to } \\
\text { improve the or- } \\
\text { ganization pro- } \\
\text { cess }\end{array}$ & & $\bullet$ & & \\
\hline $\begin{array}{l}\text { Using scientific } \\
\text { support and re- } \\
\text { search team to } \\
\text { improve organi- }\end{array}$ & & $\bullet$ & $\begin{array}{c}13,114,164 \\
171\end{array}$ & $142,295,254$ \\
\hline
\end{tabular}




\begin{tabular}{|c|c|c|c|c|}
\hline zation process & & & & \\
\hline $\begin{array}{l}\text { Encouraging and } \\
\text { persuading col- } \\
\text { leagues to do } \\
\text { applied research- } \\
\text { es }\end{array}$ & & $\bullet$ & $\begin{array}{c}71,98,24,48 \\
98,154,161 \\
162,169,178 \\
179\end{array}$ & $\begin{array}{c}28,53,64,107 \\
133,285,176 \\
251,291,397\end{array}$ \\
\hline $\begin{array}{l}\text { Application of } \\
\text { new research } \\
\text { projects }\end{array}$ & $\begin{array}{l}\text { Ahzab } 9 \text { Muz- } \\
\text { zammil } 19\end{array}$ & & $\begin{array}{c}3,274,98,113 \\
157\end{array}$ & $33,35,435,437$ \\
\hline $\begin{array}{l}\text { Using early re- } \\
\text { turn and applied } \\
\text { research plan }\end{array}$ & & $\bullet$ & & \\
\hline $\begin{array}{c}\text { Virtual } \\
\text { knowledge of } \\
\text { organizational } \\
\text { management }\end{array}$ & & $\bullet$ & & \\
\hline $\begin{array}{l}\text { Ability to collab- } \\
\text { orate virtually }\end{array}$ & & $\bullet$ & & \\
\hline $\begin{array}{c}\text { Using new } \\
\text { school manage- } \\
\text { ment technolo- } \\
\text { gies }\end{array}$ & & & $\begin{array}{c}416,382,82 \\
161,176\end{array}$ & $\begin{array}{c}136,143,254 \\
302,320,329,408\end{array}$ \\
\hline $\begin{array}{l}\text { Ability to work } \\
\text { with manage- } \\
\text { ment software }\end{array}$ & & $\bullet$ & & \\
\hline $\begin{array}{l}\text { Accepting the } \\
\text { criticisms of oth- } \\
\text { ers and mistakes }\end{array}$ & $\begin{array}{c}\text { Muminun } 67 \\
\text { Loqman } 18 \text { Saj- } \\
\text { da } 15 \text { Dokhan } 19 \\
\text { Najm } 32 \text { Hadid } \\
23 \text { A'raf } 186 \\
\text { R'ad } 1 \text { An'am } \\
\text { 25/9/35 Maryam } \\
97\end{array}$ & & $354,392,357$ & \\
\hline $\begin{array}{l}\text { Having the right } \\
\text { judgment power } \\
\text { in affairs }\end{array}$ & & & $\begin{array}{c}112,130,139 \\
152,161,145 \\
146,159,167 \\
173,181\end{array}$ & $224,227,163,346$ \\
\hline $\begin{array}{l}\text { Surveying col- } \\
\text { leagues about the } \\
\text { result of the im- } \\
\text { plemented pro- } \\
\text { grams }\end{array}$ & $\begin{array}{c}\text { Shooraa } 30 / 40 / \\
12 / 38\end{array}$ & & $11,141,147$ & 71,310 \\
\hline $\begin{array}{l}\text { Correct and fair } \\
\text { evaluation of } \\
\text { colleagues with- } \\
\text { out interfering } \\
\text { with personal } \\
\text { opinions }\end{array}$ & & $\bullet$ & & \\
\hline $\begin{array}{l}\text { Ability to ana- } \\
\text { lyze issues }\end{array}$ & $\begin{array}{c}\text { Yunis } 35 \text { Anbiya } \\
\text { 18/22 Haj } 17 \\
\text { Sajda } 25 \text { Saba } 26 \\
\text { Jathiyah } 17 \\
\text { Baqarah 136 Al } \\
\text { Imran }\end{array}$ & & $\begin{array}{l}198,201,204, \\
221,296,260 \\
361\end{array}$ & \\
\hline
\end{tabular}


$84 / 140 / 174 / 168 /$

$178 / 166 / 152$

An'am 52

Self-awareness

$116,142,292$,

and self-control

296, 298, 308,

$326,181,308$,

131,349

\section{Having personal- Aalaa $21 \mathrm{Hud}$ \\ ity stability $\quad 112$ Baqarah \\ $153,155,177,150$ \\ Ra'ad 24 Lo- \\ qman 17,31 \\ Ankabut 7 Hijr \\ 75 Yusuf 87 Re- \\ pentance Taw- \\ bah79 Maidah \\ $65 \mathrm{Al}$ Naas \\ 257,354}

Not creating

Anbiya 19,13

competitive and

Hud 10 Mumin-

stressful atmos-

un67 Loqman 18

phere

Sajda 15 Dokhan

19, Najm 32

Hadid 23 Nisa

53 Hujurat 4

Maidah 41,82

Anfal 25,55

Having mental Loqman 20,21

and psychologi-

cal balance

Having social Al Imran 206, 201, 240,

and emotional 103,199 Nahl 61

251, 243, 287,

intelligence

Anfal 63

390

Islamic anthro-

pological attitude

based on training

community man-

agers

The need to in-
crease the re-
sponsibility of
managers of the
organization ac-
cording to Irani-
an standards

Baqarah

$134,139,141$

Maidah 89

An'am 164 A'raf

5,135 Tawbah 3

Noor 54 Yusuf

53 Isra 36

Ankabut 12 Lo-

qman 32

\section{Using different}

views in manag-

ing affairs

\begin{tabular}{cccc} 
ing affairs & Zumar6 An'am & $66,67,119,180$, & $58,109,330,252$, \\
\hline & 98 Loqman 28 & $19,168,23,139$, & $253,254,278$, \\
princiniple of & A'raf 189 Ra'ad & $140,141,41,69$, & $280,230,347$ \\
equality & 7 Baqarah 38 & 168 &
\end{tabular}




\begin{tabular}{|c|c|c|c|c|c|}
\hline & $\begin{array}{c}\text { Jathiyah11 Mu- } \\
\text { hammad } 25 \\
\text { Qasas } 43 \text { Kahf } \\
55 \text { Ta-Ha } 23 \\
\text { Ghafir } 40 \text { Nahl } \\
97 \text { Hud } 111\end{array}$ & & & & \\
\hline $\begin{array}{l}\text { Not relying on } \\
\text { using a particular } \\
\text { opinion and point } \\
\text { of view }\end{array}$ & $\begin{array}{c}\text { Tawbah } \\
\text { 109,100,72 Nisa } \\
6 \text { Baqarah } \\
\text { 124,249,155,21 } \\
\text { Yunis } 11 \text { Hud } 7 \\
\text { Ibrahim } 6 \text { Nahl } \\
\text { 92 Saad 24,34 } \\
\text { Muhammad 31 } \\
\text { Qamar 27 Mulk } \\
\text { 2 Ghāšiyah 15 } \\
\text { Zalzalah 6 Mai- } \\
\text { dah 41,48 94 }\end{array}$ & & & $\begin{array}{c}232,233,324, \\
325\end{array}$ & \\
\hline $\begin{array}{l}\text { Having a flexible } \\
\text { intellectual hori- } \\
\text { zon }\end{array}$ & & & & $\begin{array}{c}3,23,112,241, \\
303\end{array}$ & $\begin{array}{c}2,50,65,90,99 \\
108,124,140, \\
143,150,168,263\end{array}$ \\
\hline $\begin{array}{l}\text { Using trusted } \\
\text { NGOs in } \\
\text { strengthening } \\
\text { organizational } \\
\text { affairs satisfac- } \\
\text { tion }\end{array}$ & & & $\bullet$ & & \\
\hline $\begin{array}{l}\text { Paying attention } \\
\text { to the principle } \\
\text { of humanity in } \\
\text { the field of exist- } \\
\text { ence evolutions }\end{array}$ & & & $\bullet$ & 166,169 & $160,288,403$ \\
\hline $\begin{array}{l}\text { Believing in the } \\
\text { principles and } \\
\text { worldview of } \\
\text { Islamic man- } \\
\text { agement }\end{array}$ & & $\begin{array}{l}\text { Letter } \\
27\end{array}$ & & & \\
\hline $\begin{array}{l}\text { Paying attention } \\
\text { to the administra- } \\
\text { tive rights and } \\
\text { organizational } \\
\text { affairs of manag- } \\
\text { ers in the field of } \\
\text { Islamic Iran }\end{array}$ & & & $\bullet$ & & \\
\hline $\begin{array}{l}\text { Respecting reli- } \\
\text { gious values and } \\
\text { empowering } \\
\text { managers to the } \\
\text { styles of Islamic } \\
\text { Iranian commu- } \\
\text { nication in the } \\
\text { body of the or- } \\
\text { ganization }\end{array}$ & & $\begin{array}{l}\text { Letter } \\
32\end{array}$ & $\bullet$ & & \\
\hline
\end{tabular}




\begin{tabular}{ccc}
\hline $\begin{array}{c}\text { Adherence to } \\
\text { ethical values } \\
\text { and principles in } \\
\text { the work and } \\
\text { personal field }\end{array}$ & $66,120,171$ & $56,135,262,324$, \\
$362,397,408$
\end{tabular}

In (Table 2), the themes were classified into 16 main or inclusive themes according to the common meanings and concepts among them, which include: professional competencies, individual competencies, professional ethics, personal and interpersonal and organizational communication skills, advanced organizational behavior, multicultural literacy, media literacy, computer literacy, research literacy, critical literacy, creativity and innovation, quality management, knowledge management, emotions management, attention to attitudes and values management. Finally, the inclusive themes were classified as 5 organizing themes according to their semantic and conceptual commonalities, which are: managerial competencies, behavioral competencies, skill competencies, cognitive competencies and emotional competencies.

Table 2.

Classification of Themes According to Common Meanings and Concepts among Them in the Form of 16 Main or Inclusive Themes

\begin{tabular}{|c|c|c|}
\hline $\begin{array}{l}\text { Inclusive } \\
\text { Themes }\end{array}$ & $\begin{array}{c}\text { Organizing } \\
\text { Themes }\end{array}$ & Basic Themes \\
\hline $\begin{array}{c}\text { Managerial } \\
\text { Competencies }\end{array}$ & $\begin{array}{c}\text { Professional } \\
\text { Competencies }\end{array}$ & $\begin{array}{ll}\checkmark & \text { Having expertise and experience in adopting the ap- } \\
\text { propriate management style } \\
\checkmark & \text { Management of organizational budget and credits } \\
\checkmark & \text { Ability to analyze the managerial conditions and re- } \\
\text { quirements of the organization } \\
\checkmark & \text { Intellectual capability to guide the affairs, tools and } \\
\text { environment of the organization } \\
\checkmark & \text { Management of crisis and unexpected affairs in the } \\
\text { organization } \\
\checkmark & \text { Having capable human resources to advance goals } \\
\checkmark & \text { Forming a strong advisory team to carry out part- } \\
\checkmark & \text { nership management affairs } \\
\checkmark & \text { Operational capability in using organizational pro- } \\
& \text { motion opportunities } \\
\checkmark & \text { Ability to coordinate between organizational needs } \\
\text { and demands } & \text { Professional planning for balanced development of } \\
\text { the organization } & \checkmark \text { Planning in order to select competent organizational } \\
\checkmark & \text { Ford succession forces } \\
\checkmark & \text { Fance the affairs of the organization } \\
\text { Resource optimization, design and implementation }\end{array}$ \\
\hline
\end{tabular}




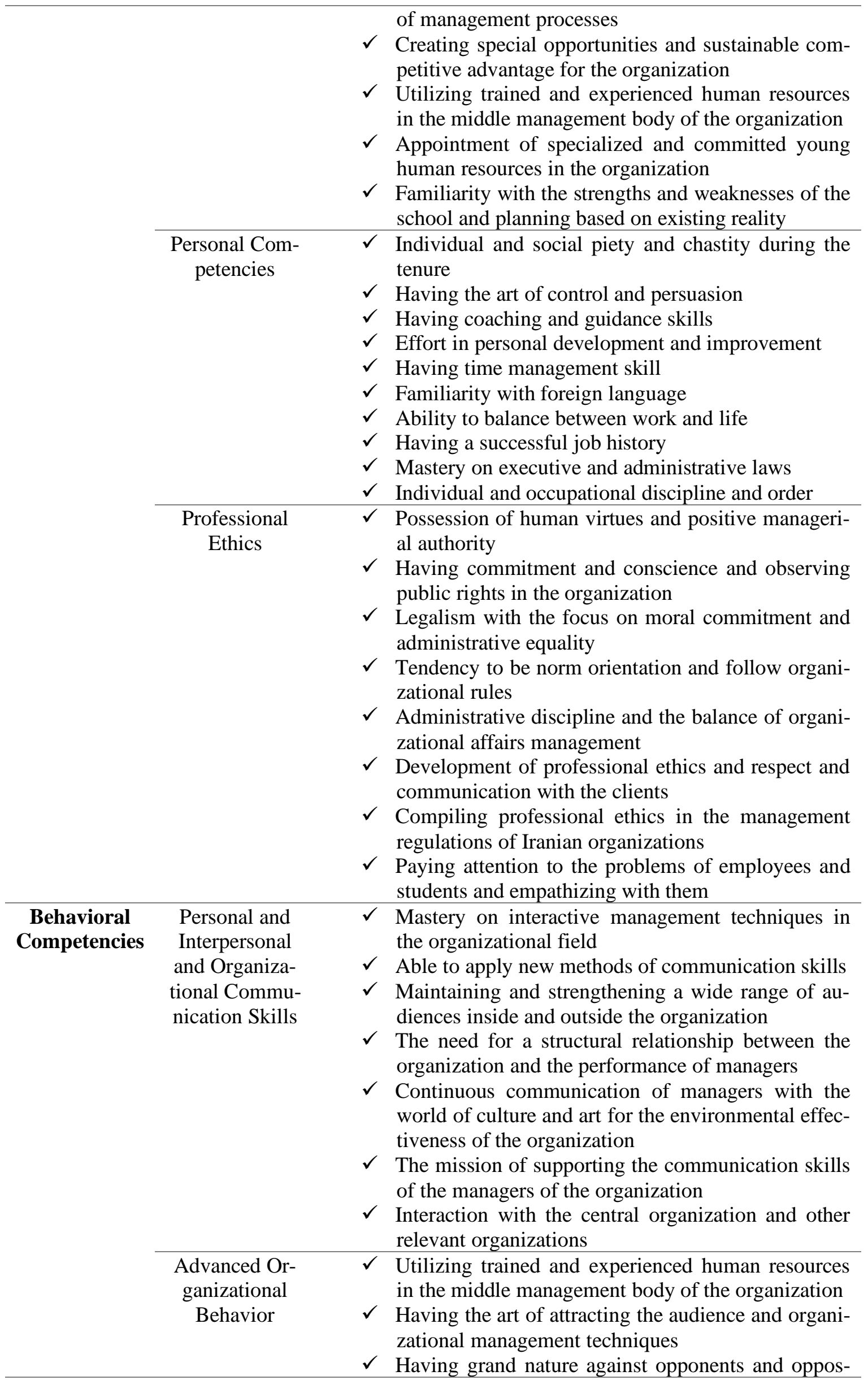




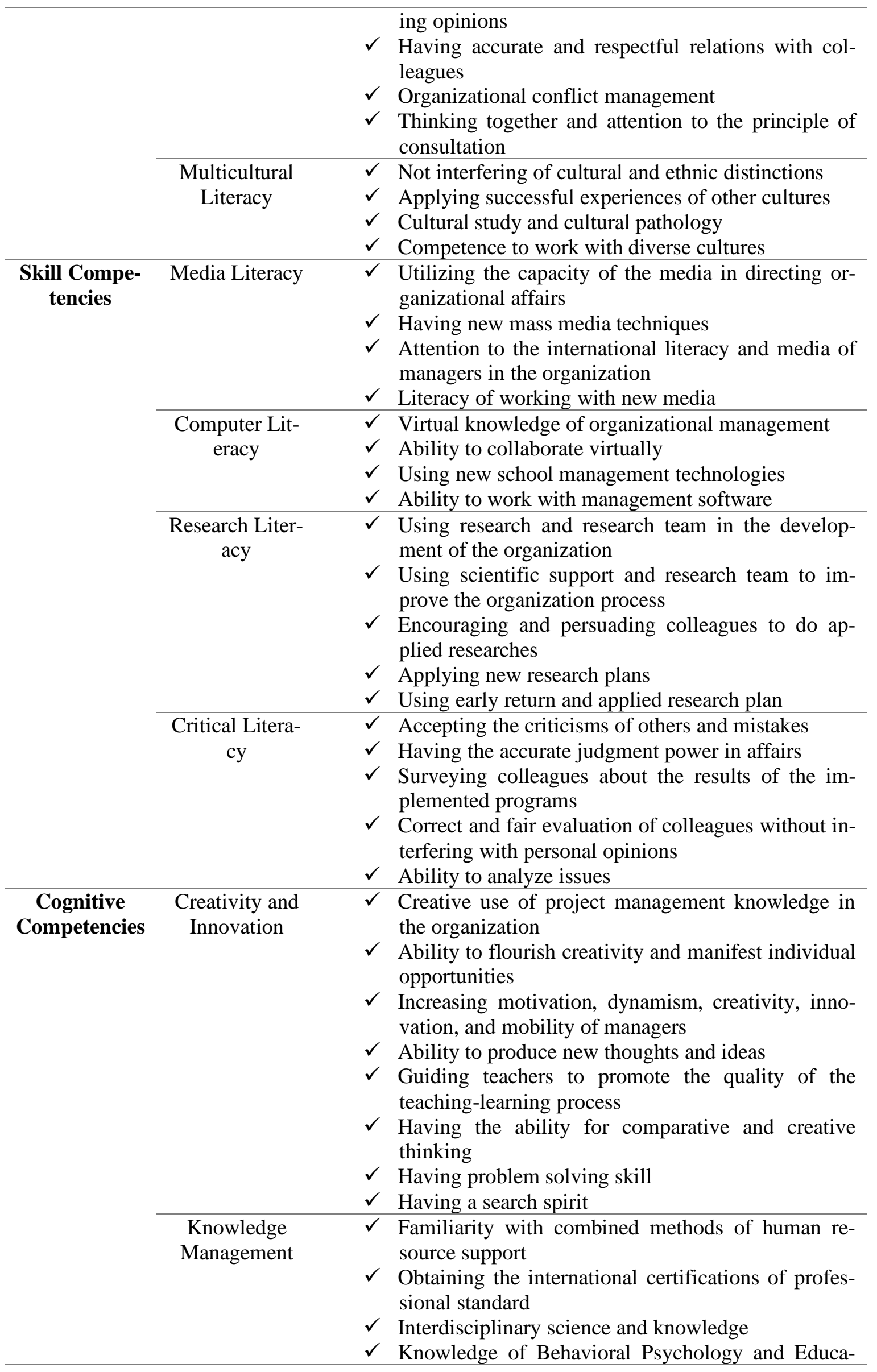




\begin{tabular}{|c|c|c|}
\hline & & $\begin{array}{ll} & \text { tional Sciences } \\
\checkmark & \text { Ability to distribute knowledge on time } \\
\checkmark & \text { Ability to encourage the creation of new knowledge } \\
\checkmark & \text { Updating knowledge resources }\end{array}$ \\
\hline & $\begin{array}{l}\text { Quality Man- } \\
\text { agement }\end{array}$ & $\begin{array}{ll}\checkmark & \text { Optimization, designing and developing middle } \\
& \text { management systems } \\
\checkmark & \text { Creating transformation } \\
\checkmark & \text { Paying attention to the quality of the facilities of the } \\
\text { educational environment } \\
\checkmark & \text { Planning for qualitative implementation of comple- } \\
\checkmark & \text { mentary extracurricular and training activities } \\
\checkmark & \text { Promoting environmental attractiveness }\end{array}$ \\
\hline \multirow[t]{3}{*}{$\begin{array}{c}\text { Emotional } \\
\text { Competencies }\end{array}$} & $\begin{array}{c}\text { Emotions Man- } \\
\text { agement }\end{array}$ & $\begin{array}{ll}\checkmark & \text { Self-awareness and self-control } \\
\checkmark & \text { Having personality stability } \\
\checkmark & \text { Not creating competitive and stressful atmosphere } \\
\checkmark & \text { Having mental and psychological balance } \\
\checkmark & \text { Having social and emotional intelligence }\end{array}$ \\
\hline & $\begin{array}{l}\text { Attention to } \\
\text { Attitudes }\end{array}$ & $\begin{array}{ll}\checkmark & \text { Islamic anthropological attitude based on the train- } \\
\text { ing the managers of community } \\
\checkmark & \text { The need to increase the responsibility of the man- } \\
\text { agers of the organization according to Iranian crite- } \\
\text { ria } \\
\checkmark & \text { Using different perspectives in managing affairs } \\
\checkmark & \text { Observing the principle of equality } \\
\checkmark & \text { Not relying on using a particular opinion and point } \\
& \text { of view } \\
\checkmark & \text { Having a flexible intellectual horizon }\end{array}$ \\
\hline & $\begin{array}{l}\text { Values Man- } \\
\text { agement }\end{array}$ & $\begin{array}{ll}\checkmark & \text { Using trusted NGOs in strengthening organizational } \\
\text { affairs satisfaction } \\
\checkmark & \text { Paying attention to the principle of humanity in the } \\
\text { field of existence evolution } \\
\checkmark & \text { Believing in the principles and worldview of Islamic } \\
\text { management } \\
\checkmark \quad \text { Paying attention to the administrative rights and or- } \\
\text { ganizational affairs of managers in the field of ad- } \\
\text { ministrating Islamic Iran } \\
\checkmark \quad \text { Respecting religious values and empowering man- } \\
\text { agers to the styles of Islamic Iranian communication } \\
\text { in the body of the organization } \\
\checkmark \quad \text { Adherence to ethical values and principles in the } \\
\checkmark \quad \text { work and personal context } \\
\checkmark \quad \text { Being popular and honoring the client } \\
\text { Organizational and individual piety and having hon- } \\
\text { esty }\end{array}$ \\
\hline
\end{tabular}

At this stage, using basic (109), inclusive (16) and organizing (5) themes obtained from interviews and written sources, the themes network related to the professional meritocracy model of high school managers of education in Bushehr province based on Islamic and Iranian teachings was drawn (Figure 1). 


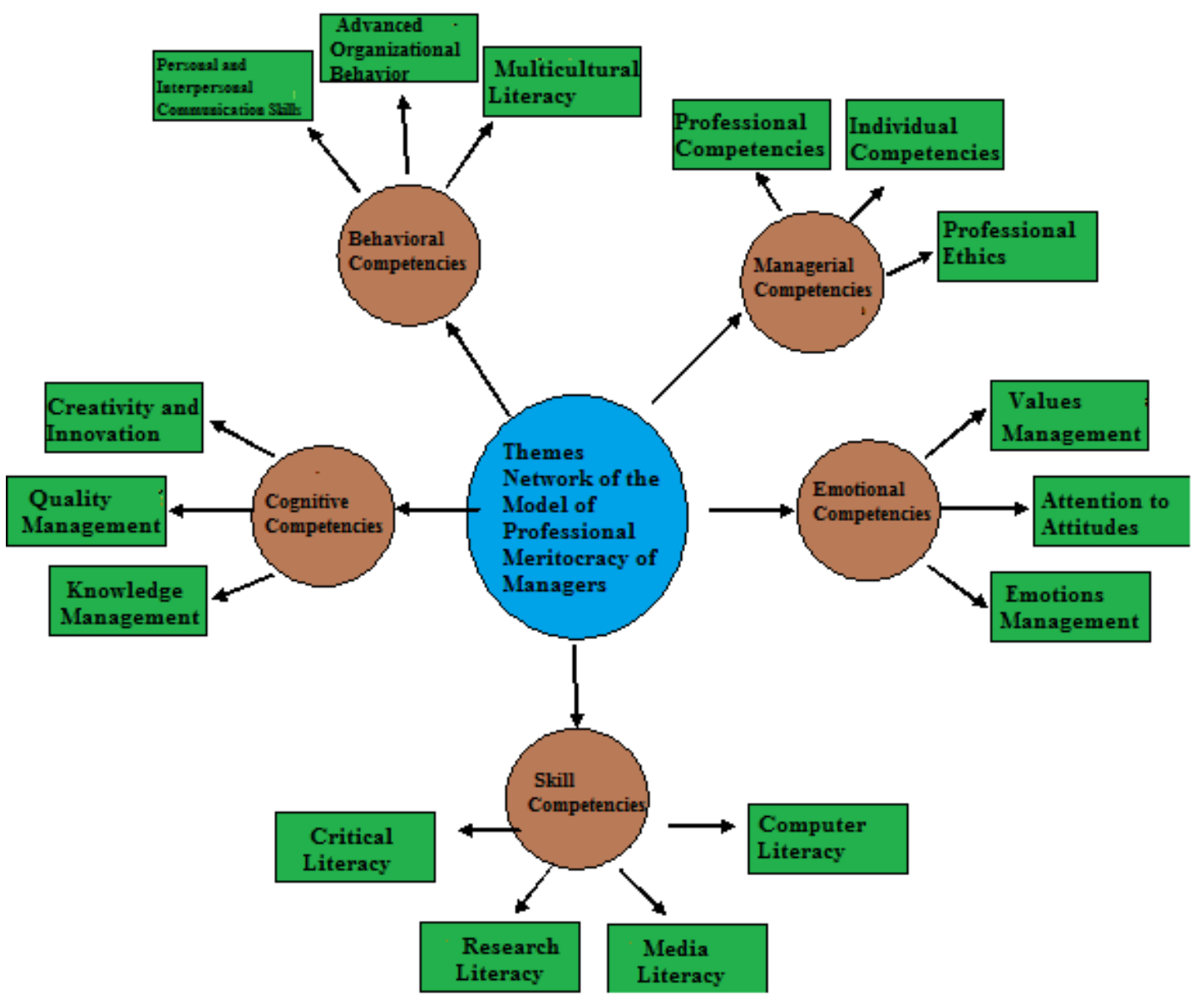

Figure 1. Themes Network of the Model of Professional Meritocracy of the Educational Managers of High School Based on Islamic-Iranian Teachings 


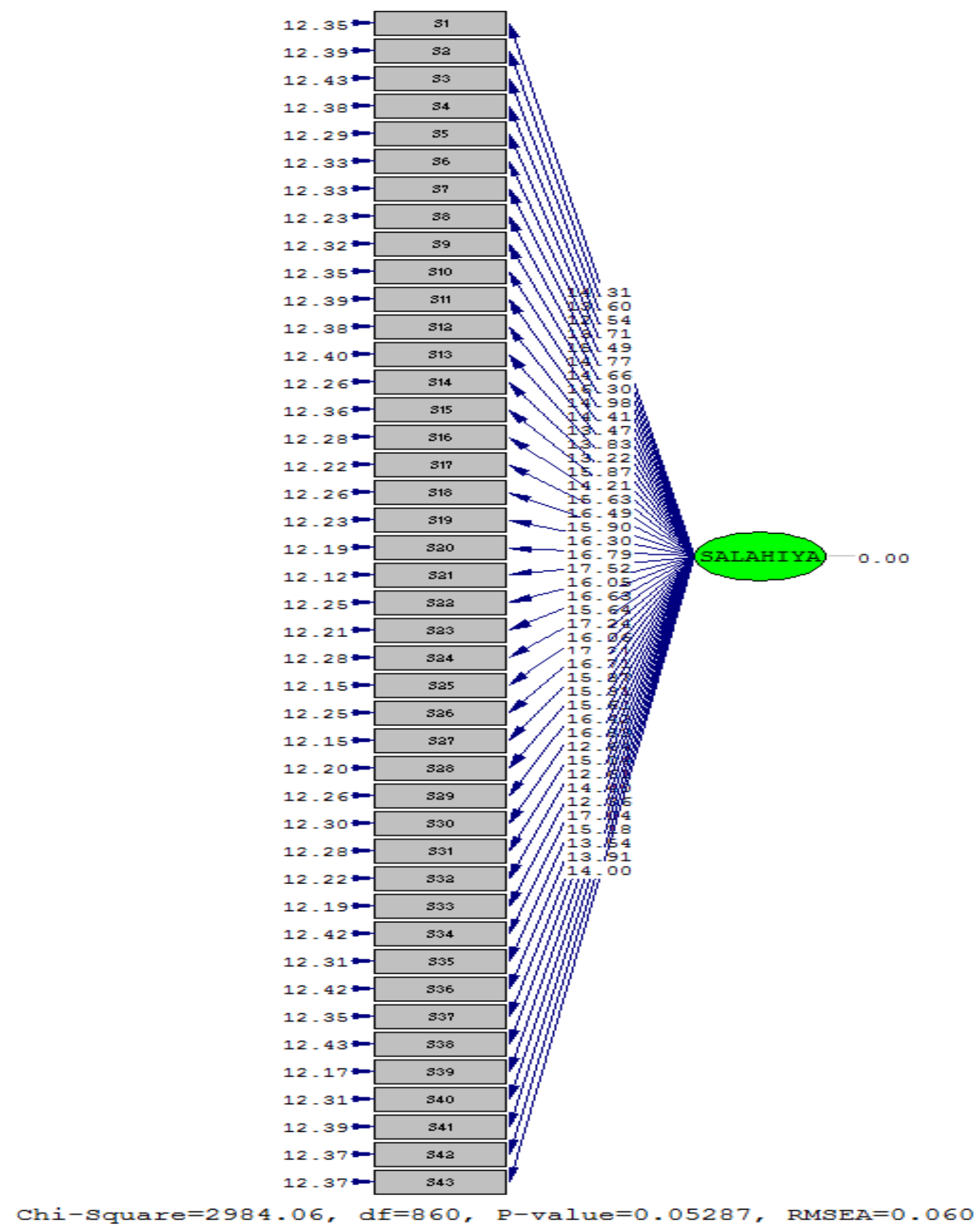

Figure 2. Model of Professional Competence of Managers in the Significance Coefficients State

The data normality related to the research variables was investigated and confirmed using the Kolmogorov-Smirnov test (Figure 2)..

Descriptive Analysis of the Demographic Variables of Quantitative Section

In this section, demographic information of the subjects has been described based on gender and the level of education. In this section, the subjects were classified according to gender, that the (Diagram 1) shows that $47 \%$ of the subjects are female and $53 \%$ are male. 


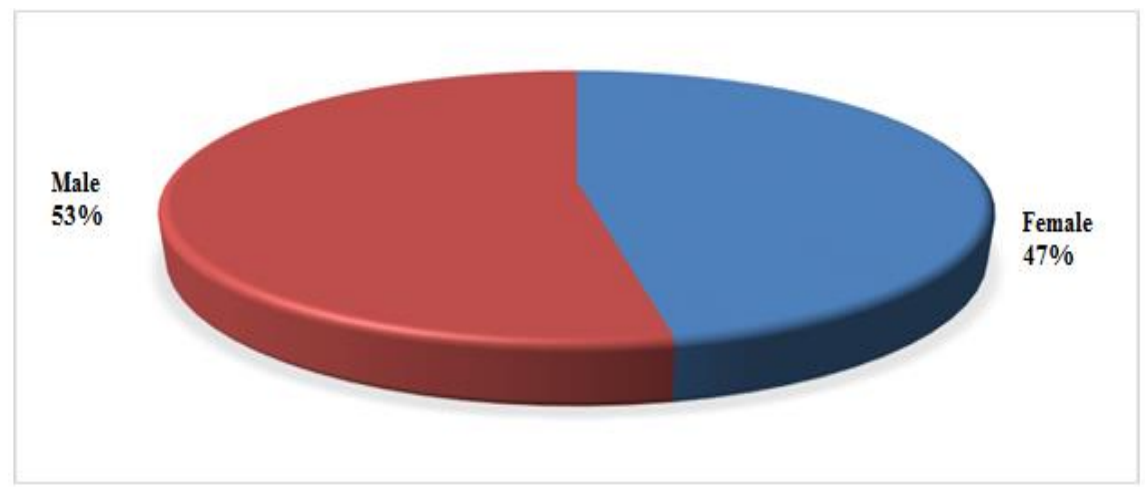

Diagram 1. Relative Distribution of Subjects' Gender

The Chi-square and RMSEA indicators show that the model provides a more proper fit to the data. The model outputs have been investigated in the (Table 3) and (Table 4).

Table 3.

Fit Indicators of Model Path Analysis - Internal Factors Affecting Professional Competence

\begin{tabular}{lcc}
\multicolumn{1}{c}{ Index Name } & \multicolumn{2}{c}{ Fit Indicators } \\
\cline { 2 - 3 } & Value & Permitted Limit \\
\hline Chi-square/df & 2.68 & Lower than 3 \\
\hline $\begin{array}{l}\text { Root Mean Square Error of Approximation } \\
\text { (RMSEA) }\end{array}$ & 0.063 & Lower than 0.1 \\
\hline Comparative Fit Index (CFI) & 0.98 & Higher than 0.9 \\
\hline Normed Fit Index (NFI) & 0.97 & Higher than 0.9 \\
\hline Goodness of Fit Index (GFI) & 0.95 & Higher than 0.9 \\
\hline Adjusted Goodness of Fit Index (AGFI) & 0.93 & Higher than 0.9 \\
\hline
\end{tabular}

Table 4.

Fit Indicators of Model Path Analysis - External Factors Affecting Professional Competence

\begin{tabular}{lcc}
\hline \multicolumn{1}{l}{ Index Name } & \multicolumn{2}{c}{ Fit Indexes } \\
\cline { 2 - 3 } & Value & Permitted Limit \\
\hline Chi-square/df & 2.88 & Lower than 3 \\
\hline $\begin{array}{l}\text { Root Mean Square Error of Approximation } \\
\text { (RMSEA) }\end{array}$ & 0.067 & Lower than 0.1 \\
\hline Comparative Fit Index (CFI) & 0.98 & Higher than 0.9 \\
\hline Normed Fit Index (NFI) & 0.97 & Higher than 0.9 \\
\hline Goodness of Fit Index (GFI) & 0.93 & Higher than 0.9 \\
\hline Adjusted Goodness of Fit Index (AGFI) & 0.91 & Higher than 0.9 \\
\hline
\end{tabular}

As it is observed, the pattern fit indicators have been located in a desirable situation.

\section{Discussion}

From the perspective of the teachings and resources of ancient Iran until now, the issue of meritocracy and the use of efficient forces have been considered as one of the basic principles and among the factors for the success of organizations in moving towards dynamism and evolution. In other words, meritocracy is as the key to the success of developmental movements within organizations; in a way that managers under no circumstances should consider themselves needless to pay attention to its concepts and teachings. Paying attention to providing the desirable 
model of organizational management and compiling an operational model and drawing a roadmap can lead the organization to excellent goals with better order and program, and Islamic and Iranian teachings consideration in compiling a model of meritocracy of organizational managers, promises a clear vision of organizational progress and excellence. Considering the pivotal role of human resource professional competencies in managerial tendencies, the present research has recognized and removes traditional ambiguities in this field and has tried to provide a model with validity and reliability confirmed by subject matter experts that can meet the needs of competent managers. The following results were obtained according to the method of theme analysis and combined findings. In the qualitative section: components composing professional competence include five organizing themes including cognitive competencies ( 3 themes), skill competencies (4 themes), emotional competencies ( 3 themes), behavioral competencies ( 3 themes) and managerial competencies ( 3 themes). In the quantitative section it includes internal and external influential components on the professional competence of educational managers, which include the components of individual characteristics (10 items), structure (6 items), management (9 items), information technology (9 items), as internal components, and the components of healthy society (9 items), media ( 9 items) and educational policies of the environment and parents (8 items), as the most important indicators identified were proposed and identified in the field of meritorious management components from the perspective of Islamic and Iranian teachings that are: commitment and work conscience, thoughtfulness, professionalism, meritocracy, legalism, justice seeking, open-mindedness, humility, consultability, trustworthiness, commitment to organizational principles and avoidance of unfavorable human traits. Meritocracy requires the provision of relevant infrastructures in terms of structure, attitude, technology, law and organization. The results of the above findings are consistent and in line with the results of (Niknami \& Karimi, 2009) and (Abdullahi et al., 2014) in terms of method and purpose. For the network of professional meritocracy themes for managers from the perspective of IslamicIranian teachings by the themes analysis approach, at first the basic themes, then the inclusive themes and finally the organizing themes were identified and the themes network was drawn. The elements of each component were identified as the primary themes. The most important theme of competent management and the most valid criterion for analyzing its effectiveness from the perspective of Islamic-Iranian teachings with a theme analysis approach is that the managers of any organization have the necessary behavioral characteristics, skills and managerial abilities required to acquire identity from the beginning of responsibility and during the management time. The ability of the manager to use the intellectual and social capital of the competent people of the society within the organizations in order to develop the society is one of the most important thematic elements of meritocratic management from the perspective of Islamic-Iranian teachings. The results of the above findings are consistent and in line with the results of (Ellen et al., 2020) and also with the results of (Almasi, 2018) in terms of method and purpose. Regarding the professional meritocracy framework for managers from the perspective of Islamic-Iranian teachings with a theme analysis approach, it can be said that the meritocratic management framework of the organization is set based on the "human systems management" framework and is required for optimal management of all organizations. Organizational framework used in this research is designed and compiled based on the goal of this paper after studying various models of managers' competencies and investigating the provided dimensions, components, and indicators provided in this model. According to the results of theoretical studies and qualitative research and the method of interviewing subject matter experts, the competency framework of the managers of the organization, including 34 various key competencies in three layers of managerial competencies, behavioral competencies and professional competencies and seven groups of individual, perceptual, organizational, financial, leadership and executive competencies, can be designed and proposed. This framework has two dimensions of general competencies and specialized competencies based on managerial roles, public relations, media communication and intra-organizational communication. The general competencies dimension includes 3 components of human, personal and value competencies and the specialized competencies dimension has been classified into 5 components of technical, perceptual, professional ethics and professional competencies. The results of the above findings in terms of method and 
purpose are consistent and in line with the results of (Maleki, 2005) and (Namdar, 2010) as well as the results of (Deloub \& Marion, 2005).

\section{Conclusion}

The validity of the professional meritocracy model for managers from the perspective of Islamic-Iranian teachings, five general components and categories in the form of a paradigm model including individual influential conditions and factors, organizational conditions and factors, situation and conditions of the organization, performance improvement strategy of the members of organization and the results of implementing programs with sub-components have led to the validation of the professional meritocratic management model for managers from the perspective of Islamic-Iranian teachings. Having formed the initial framework of the professional meritocracy operational model for managers from the perspective of Islamic-Iranian teachings by the theme analysis method, qualitative validation criteria were used to assess the validity of the initial framework. Reliability test was used to ensure the qualitative reliability of the initial framework. Managers' competencies have been identified in the five main clusters of intellectual abilities, functional and executive characteristics, knowledge and awareness, communication and interpersonal skills, and personality traits and titles; adherence to principles, values and strategic thinking, responsibility, creativity in pursuit and consequentialism, team management, problem solving, networking, analytical-systemic thinking, flexibility, and financial understanding. The results of the above findings are consistent and in line with the results of Blasco, Matuska, Rosac (Shah Pasand, 2008) and (Armstrong, 2006) and (Lee et al., 2011) and (Sundberg \& Wallin, 2005) the results of (Loghmani Shahmiri et al., 2015).

Finally, it is suggested that an arrangement be made that in assigning and appointing organizational managers, the criterion of intellectual and practical merit should be given the first priority in scoring and effective assessment criteria should be used instead of friendly relations. It is suggested to future researchers to design an applied and executive model of competency based on Islamic and Iranian teachings in order to institutionalize meritocracy and promote the general performance of managers. The main limitation of this research was that managers, despite being aware of the criteria of professional competencies of managers, unfortunately do not consider these criteria in their appointments.

\section{References}

Feldman D. \& Morinio D. (2018), Academic Leadership: A practical Guide to Chaining the Department, 2nd Ed. New York: Anker Publishing Company, 13(49): 25-48.

Soderquist S. (2010), "The convergence of National Professional Qualifications in educational leadership and master's level study", Higher Education, Skills and Work-Based Learning, 8(4), 484-494.

Dragandis D. \& Mentras B. (2006), The relationships between supplier development, commitment, social capital accumulation and performance improvement, Journal of Operations Management, 25(6): 528-545.

Donaldson J. \& Davis P. (1990), Business ethics? Yes, but what can it do for the bottom line?. Management Decision, 25(6): 528-545.

Fathi Vajargah K. \& Mohammad Hadi F. (2013), "Fundamentals of Quality Management in Training and Improving Human Resources", Tehran: Publication of Abij, 9(3): 38-55.

Soleimani A. \& Karimi M. (2016), "Assessing the Competencies and Skills Required by Teachers in the Professional Development of Interns and Trainees of Farhangian University", Conference on Internship, Entrepreneurship and Professional Competencies Development, Farhangian University, 26(6): 44-52.

Ghirshman R. (2016), "Iran from the Beginning to Islam", Publication of Negah, 11(5): 174200. 
Mohammadi Mehr M. \& Maleki H. \& Abbaspour A. \& Khoshdel A. (2013), "A Survey on the Competencies Required For Lifelong Learning in General Medicine Students", Iranian Journal of Education in Medical Sciences, 11(8): 903-916.

Geejung H. \& Keelee D. (2020), Service productivity towards a conceptualization of the transformation of inputs into economic results in services. Journal of Business Research, 5(7): 414-423.

Group S. (2003), Labor productivity Growth, Education: Health and Technological progress: A cross- country Analysis, Economic Analysis \& policy, 40(2): 249-253.

Kimmer P. \& Lambrechts W. \& Verhulst E. \& Rymenams S. (2017), Professional development of sustainability competences in higher education: the role of empowerment. International Journal of Sustainability in Higher Education, in press, psychology, 3(2): 77-101.

Sobhaninejad M. \& Tejdan A.(2015), "Evaluating the Competencies of Instructors and Masters of "Training along with Production" outside the Knowledge Work Center", Educational Researches, 30(5): 24-64.

Abedi Jafari H. \& Taslimi M. \& Faqihi H. \& Sheikhzadeh M. (2011), "Theme Analysis and Themes Network: A Simple and Efficient Way to Explain Patterns Available in Qualitative Data", Strategic Management Thought, 5(2): 151-198.

Niknami M. \& Karimi F. (2009), "Professional Competencies of General Education Teachers and Providing An Appropriate Perceptual Framework", Journal of Knowledge and Research in Educational Sciences and Curriculum, Isfahan: Islamic Azad University, Khorasgan Branch, 15(3): 84-96.

Abdullahi B. \& Dadjooy Tavakoli A. \& Youseliani G. (2014), "Identifying and Validating the Professional Competencies of Effective Teachers", Quarterly Journal of Educational Innovations, $13(49)$ : 25-48.

Ellen D. \& Annie H. \& Jan H. (2020), Exploring the outcomes of group reflective learning for school leaders Received 21 Mar 2020, Accepted 16 Jun 2020, Published online, 15(3): 84-96.

Almasi M. (2018), "History of Islam and Iran Education", Publication of International Publishing and Printing Company, Affiliated to the Islamic Development Organization, 15(3): 84-96.

Maleki H. (2005), "Professional Competencies of Teaching" Tehran: Publication of Madreseh, 15(3): 84-96.

Namdar R. (2010), "Professional Competencies Needed by Evaluation Experts of the Ministry of Jihad Agriculture", Quarterly Journal of Agricultural Education Management Research, 12(8): 22-31.

Deloub J. \& Marion R. (2005), Higher education leadership roles in knowledge processing, The Learning Organization, 12(2): 140-151.

Shah Pasand M. (2008), "Determining the Components of the Process of Professional Development of the Trainers of the Training Centers of the Ministry of Jihad Agriculture", Research and Construction in Agriculture and Horticulture, 8(1): 131-139.

Armstrong D. (2006), A WORD of state of Affairs, Cambridge: Cambridge university press, 11(2): 107-120.

Lee T. \& Kim D. \& Lee D. (2011), A competency model for project construction team and project control team. KSCE Journal of Civil Engineering, 15(5): 781-792.

Sundberg H. \& Wallin P. (2005), "Icebergs drifting a part - customer strategies affecting systems development and information management" in proceedings of the IADIS, international conference e-society 2005, Qawra, Malta, 14(5): 619-624.

Loghmani Shahmiri M. \& Hosseini Khalili S. \& Sadati J. \& Bamati Tusi R. (2015), "Investigating the Professional Competencies of Teachers", Third Scientific Research Conference on Educational Sciences and Psychology of Social and Cultural Damages in Iran, 9(4): 24-64. 